\title{
Evaluation of Economic Importance of Locally Produced Manure over Inorganic Fertilizer for Maize Production: Vegetative Performance and Cost Implication
}

\author{
Fatima Aliyu Deba', Safiya Isma'il' ${ }^{1}$, Muhammed Rabiu Sahal'1, Ibrahim Yusuf Okpanachi' \\ ${ }^{1}$ Abubakar Tafawa Balewa University, Bauchi, Nigeria \\ ${ }^{2}$ University of Lagos, Lagos, Nigeria \\ Email: fatimadeba2018@gmail.com
}

How to cite this paper: Deba, F.A., Isma'il, S., Sahal, M.R. and Okpanachi, I.Y. (2019) Evaluation of Economic Importance of Locally Produced Manure over Inorganic Fertilizer for Maize Production: Vegetative Performance and Cost Implication. American Journal of Molecular Biology, 9, 64-74. https://doi.org/10.4236/ajmb.2019.92006

Received: December 13, 2018

Accepted: April 25, 2019

Published: April 28, 2019

Copyright $\odot 2019$ by author(s) and Scientific Research Publishing Inc. This work is licensed under the Creative Commons Attribution International License (CC BY 4.0).

http://creativecommons.org/licenses/by/4.0/

cC) (7) Open Access

\begin{abstract}
Background: Crops of economic importance like Maize are preferred to other crops with capacity of giving the highest yields, having the ability to help alleviate poverty within the African continent. The consistent use of inorganic fertilizers has great adverse effect on soil structure. The quest to seek alternative method of boosting maize production is imminent to avoid further soil degradation. Most plants form symbiotic relationships with mycorrhizae and improve nutrients uptake by host plants. Objectives: The aim of this study is to examine the effect of mycorrhizae in local manure production on development of maize, in comparison with inorganic fertilizer application and its cost implication. Methods: The experiment involved samples of starter soil from 7 local governments. Organic waste was added to starter soil, each having three replicates. Nine other replicates served as non-inoculated controls and nine served for fertilizer application at $50 \mathrm{~g}, 100 \mathrm{~g}$ and $150 \mathrm{~g}$. One [1] gram of Maize sown in $50 \mathrm{ml}$ pot contains soil at different treatment levels 50 g, $100 \mathrm{~g}$, and $150 \mathrm{~g}$. After two weeks, samples were transplanted in an open field. Results: The result showed plots treated with locally produced fertilizer, significantly influenced plant height, culm diameter, number of leaves and leaf broadness in comparison to inorganic fertilizer, which showed 53\%, 47\% respectively. Conclusion: Cost production of a bag of organic fertilizer was one-fourth cheaper compared to an inorganic fertilizer price from an open market.
\end{abstract}

\section{Keywords}

Mycorrhizae, Organic Fertilizer, Inorganic Fertilizer, Maize Production 


\section{Introduction}

Maize is an economic plant, with an impacting capacity to surprise humans. It is a staple food crop grown in diverse environments and consumed by people of varying preference and socio-economic background in Africa. Maize is one of the crops that give the highest yield per man hour of labor spent on it; it provides nutrients in compact form [1]. In terms of taste, many people prefer maize to their local cereals [1]. Importance of maize cannot be over-emphasized in the developing world, including the potential to mitigate the present food insecurity and alleviate poverty. Maize is preferred staple food for over 900 million poor consumers, 120 - 140 million poor farm families and about one third of malnourished children [2]. Due to the fact that maize is highly responsive to production inputs, its food and industrial uses are many and its production potential can hardly be matched by any other cereals. It is therefore definitely a solution to hunger.

Maize is one of the crops that depend substantially on nitrogen for growth and development. It has been established that among all the essential nutrients, nitrogen seems to have the most pronounced effect on plant growth and development [3]. As importance as nitrogen is in plant life, it is low in soils of Nigerian Guinea Savanna. The soils of this zone are characterized with low organic carbon, total nitrogen, available phosphorus, effective Cation Exchange Capacity (CEC) and exchangeable cations as well as clay and silt contents [4]. It has been observed that nitrogen is the most important constraint to increased maize production in the Guinea Savanna of Nigeria [5]. It should be noted the growth and productivity of any plant rest on degree of effectiveness of such a plant to absorb and mobilize the nutrients that are available in the soil for plant growth and dry matter accumulation [6]. Hence, application of nitrogenous fertilizer becomes inevitable to guarantee increased maize production for maximum yield. Long term use of inorganic fertilizer causes deleterious effects on soil health and environment [7]. This has led to seeking alternate source of mineral enhancement in soil, shifting attention to locally produced manure.

Arbuscular Mycorrhizae (AM) plays a crucial role in plant nutrient uptake, water relations, ecosystem establishment, plant diversity, and the productivity of plants. Mycorrhizae send out extensive networks of fine thread which facilitate uptake of limiting nutrients to include phosphorous, nitrogen, several micronutrients to the plant [8] and are capable of absorbing inorganic $\mathrm{P}$ either from the soluble $\mathrm{P}$ pools in the soil or from insoluble forms such as rock phosphates via localized $\mathrm{pH}$ alterations or by producing organic acid anions which act as chelating agents [9].

Furthermore, AM fungi can also have a direct effect on the ecosystem, as they improve the soil structure and aggregation and drive the structure of plant communities and productivity [10] [11] [12].

This research is focused to evaluate the effect of locally made manure induced with mycorrhizae on development of maize, to compare with inorganic fertilizer 
effect and its cost implication.

\section{Materials and Methods}

\subsection{Collection of Starter Soil}

Starter soils were collected in Nigeria from Bauchi State within seven local governments in an open field described by Bauchi State Agricultural Development Program (BSADP) all within Bauchi metropolis namely; Dass (M1), Giade (M2), Tafawa Balewa (M3), Darazo (M4), Ganjuwa (M5), Jama'are (M6) and Bauchi (M7).

Twenty-Five gram of soil samples containing mycorrhiza fungi was obtained using a hand shovel and placed in a polythene bag from each local government and transported to the laboratory between the Month of May-August 2018.

\subsection{Identification of AM Fungi}

Identification to genus level will usually be sufficient to determine whether or not a specimen is mycorrhizal. Identification of AM fungi was carried out in two ways;

\subsubsection{Macroscopic Identification}

At first level, soil specimens from an open field were collected and accurately described using colours chart of "the Mathew handbook of colour" [13].

\subsubsection{Microscopic Identification}

Total heterotrophic count was made after incubation at room temperature with some fungi observed with distinctive features while others lacks key of identification from the information and photographs in field guide. Thus, a second level of investigation was often required to accurately identified species of mycorrhizae fungi. This involved examination of microscopic character such as isolation of fungi into pure culture and consulting specialist for identification by wet preparation and observing the hyphae and spores within the sporangia [14].

\subsection{Mycorrhiza Multiplication}

Twenty grams of soil samples from each local government are poured into a $25 \mathrm{~L}$ container, containing 2L of microbial growth enhancer (molasses), aerator placed within the closed $25 \mathrm{~L}$ for proper mixing and left for about $4-5$ days as described by [15].

\subsection{Quantification of Mycorrhiza}

Two Milliliter of the starter substrate was scooped from the surface of mixed samples for mycorrhiza multiplication and poured into test-tube to. Five Milliliter of of trypan blue was added then filled with distilled water to $25 \mathrm{ml}$ mark. The $25 \mathrm{ml}$ of sample was centrifuged at $1000 \mathrm{rpm}$ for 5 mins [16].

Then decanted samples was viewed and counted using hemocytometer count as described by [17].

Cells per $\mathrm{ml}=$ the average count per square $\times$ the dilution factor $\times 104$ (count 
10 squares)

Total cell number $=$ cells per $\mathrm{ml} \times$ the original volume of fluid from which cell sample was removed.

\subsection{Local Manure Production}

Manure production was carried out as described by [15]. $25 \mathrm{~kg}$ of chicken dunk was mixed with the $25 \mathrm{~L}$ content and mixed with $10 \mathrm{~kg}$ of soil. The mixture is left for the period of 2 - 3 weeks in a moist condition to enable mycorrhiza extension.

\subsection{Growing of Maize Seeds Using Produces Manure}

Two-third (2/3) of Normal soils were introduced to the various pots having $1 / 3$ of organic manure introduced. The seed were sown and nurtured for 2 weeks before transplanted in an open filed using a $(2 \times 2)$ meter complete block design [16].

\subsection{Application of Fertilizers}

Mycorrhizae induced organic fertilizer and inorganic fertilizer was applied early morning hours between 6.00 am - 7.00 am. Mycorrhizae induced organic fertilizer was applied at the initial stage at $50 \mathrm{~g}, 100 \mathrm{~g}$ and $150 \mathrm{~g}$ and applied on the field at the $5^{\text {th }}$ week. Inorganic fertilizer was applied twice on the $5^{\text {th }}$ and $8^{\text {th }}$ week at $50 \mathrm{~g}, 100 \mathrm{~g}$ and $150 \mathrm{~g}$.

\subsection{Recording of Growth Parameters}

Growth characters recorded are; Plant height was measured in centimeter using a meter rule starting from the surface of the soil to the tip of the flag leaf, number of leaves were counted on the plant, Culm diameter was measured in centimeter using a thread placed round the mid portion of the culm, and then placed on a ruler and leaf broadness was measured in centimeter using a meter rule taking the leaf width.

\section{Results and Discussion}

Generally, across station spore count range from $1.6 \times 10^{7}-3.8 \times 10^{7}$. M6 (Jama'are) had the highest sporulation and the least was observed in M7 (Bauchi). Abundance of spore within soil environment could be as a result of land formation, topography, soil content and level of anthropogenic activities within sampling points as shown in Table 1.

The effect of organic and inorganic fertilizer on plant height for 5th - 9th week. At 5th week mycorrhiza induce organic fertilizer M6T2 had the highest at $81.67 \mathrm{~cm}$ and inorganic fertilizer F1T3 had the least at $51 \mathrm{~cm}, 6$ th week organic fertilizer had the highest growth M6T2 at $105.67 \mathrm{~cm}$ and inorganic fertilizer had the least growth F1T3 at $65 \mathrm{~cm}$, 7th week organic fertilizer had the highest growth M4T2 at $147 \mathrm{~cm}$, both organic, inorganic M3T1, F1T3 had the least 
Table 1. Spore counts of prepared sample gotten from different local government in Bauchi state.

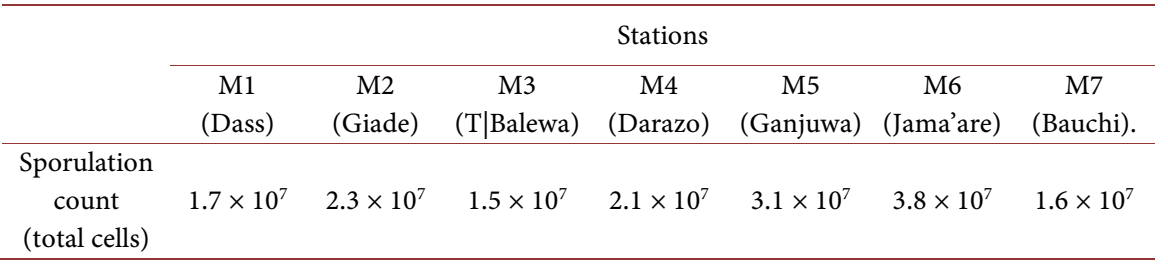

growth at $93 \mathrm{~cm}, 97 \mathrm{~cm}$. At 8th week organic fertilizer had the highest plant height at $170 \mathrm{~cm}$ and both organic, inorganic treatments M3T1, F1T3 had the least plant height at $96.33 \mathrm{~cm}$ and $109.67 \mathrm{~cm}$. 9th week organic fertilizer induced with mycorrhizae M5T2 had the highest plant height and both organic and inorganic had the least $102 \mathrm{~cm}, 117.67 \mathrm{~cm}$. 5th, 6th and 7th week showed significant difference between replicates and treatments at $(\mathrm{p}<0.05=0.047,0.156$, $0.017 ; 0.000,0.011,0.001)$ as shown in Table 2 . It showed no significant difference between replicates at ( $p>0.05=0.290,0.601$ ), but was significant between treatments at $(\mathrm{p}<0.05=0.000)$ in 8th and 9th week.

The effect of organic and inorganic fertilizer on number of leaves was observed in Table 3 for 5th - 9th week. At 5th week mycorrhiza induce organic fertilizer M3T1 had the highest number of leaves at 9.00, both organic and inorganic fertilizer M2T2, F1T3 had the least at 6.00, 6th week organic fertilizer had the highest number of leaves M2T1 at 12 and inorganic fertilizer had the least number F1T3 at $7 \mathrm{~cm}$, at 7 th week organic fertilizer had the highest growth M3T2 at 12, organic M7T1, M2T1, M2T3 had the least number of leaves at $8 \mathrm{~cm}$. 5th, 6th and 7th week showed no significant difference between replicates at $(\mathrm{p}>$ $0.05=0.739,0.405,0.920)$ and between treatment showed significant difference at $(\mathrm{p}<0.05=0.006,0.000,0.081)$. At 8th and 9th week showed no significant difference at $(\mathrm{p}>0.05=0.896,0.337)$. Organic fertilizer induced with mycorrhizae M5T1 had the highest number of leaves 11 and both organic and inorganic had the least 7.

The effect of organic and inorganic fertilizer on number of culm was shown in Table 4 from 5th - 9th week showed no significant difference between replicates and treatments at $(\mathrm{p}<0.05=0.103,0.665,0.261,0.183 ; 0.235,0.743,0.258$, 0.162) of organic fertilizer induced with mycorrhizae and inorganic fertilizer.

Table 5 shows the effect of organic and inorganic fertilizer on Leaf broadness from 5th - 9th week. At 5th, 6th week, showed no significant difference between replicates at $(p>0.05=0.581,0.515)$ and between treatments was significant at ( $p<0.05=0.005,0.000)$, but was significant in 7 th week between replicates and treatment at $(\mathrm{p}<0.05=0.012,0.04)$ and showed no significance in 8th and 9th week at $(\mathrm{p}<0.05=0.245,0.364)$ of organic fertilizer induced with mycorrhizae and inorganic fertilizer.

The mycorrhiza induced organic fertilizer showed 53\% growth rate compared to inorganic fertilizer application at $47 \%$ as clearly observed in Figure 1. 
Table 2. Average mean of plant height $[\mathrm{cm}]$ from $5^{\text {th }}-9^{\text {th }}$ week of observation.

\begin{tabular}{|c|c|c|c|c|c|}
\hline & & Weeks & & & \\
\hline Treatments & $5^{\text {th }}$ & $6^{\text {th }}$ & $7^{\text {th }}$ & $8^{\text {th }}$ & 9th \\
\hline M1T1 & 71.333abcd & $87.000 \mathrm{ab}$ & $135.667 \mathrm{ab}$ & $156.333 \mathrm{abcd}$ & $169.000 \mathrm{abcd}$ \\
\hline M1T2 & $60.333 \mathrm{bcde}$ & $88.667 \mathrm{ab}$ & $126.000 \mathrm{ab}$ & 131.000abcde & 141.333abcde \\
\hline M1T3 & 70.067abcde & $89.333 \mathrm{ab}$ & $136.000 \mathrm{ab}$ & $159.333 \mathrm{ab}$ & $172.333 \mathrm{abc}$ \\
\hline M2T1 & 70.333abcde & $90.333 \mathrm{ab}$ & $101.000 \mathrm{ab}$ & $111.667 \mathrm{cde}$ & $122.000 \mathrm{bcde}$ \\
\hline M2T2 & $70.667 \mathrm{abcd}$ & $91.000 \mathrm{ab}$ & $132.000 \mathrm{ab}$ & $146.000 \mathrm{abcd}$ & 158.333abcd \\
\hline M2T3 & $75.133 \mathrm{abc}$ & $85.000 \mathrm{ab}$ & $112.000 \mathrm{ab}$ & 127.333abcde & 139.667abcde \\
\hline M3T1 & $73.667 \mathrm{abc}$ & $79.000 \mathrm{ab}$ & $93.333 b$ & $96.333 \mathrm{e}$ & $102.000 \mathrm{e}$ \\
\hline M3T2 & $76.733 \mathrm{ab}$ & $90.000 \mathrm{ab}$ & $104.333 \mathrm{ab}$ & $116.667 \mathrm{bcde}$ & 129.667abcde \\
\hline M3T3 & 69.333abcde & $97.000 \mathrm{ab}$ & $116.667 \mathrm{ab}$ & $134.000 \mathrm{abcde}$ & 146.667abcde \\
\hline M4T1 & 66.500abcde & $86.667 \mathrm{ab}$ & $136.000 \mathrm{ab}$ & $165.667 \mathrm{a}$ & $175.000 \mathrm{a}$ \\
\hline M4T2 & 66.667abcde & $87.667 \mathrm{ab}$ & $147.000 \mathrm{a}$ & $170.000 \mathrm{a}$ & $181.667 \mathrm{a}$ \\
\hline M4T3 & 70.333abcde & $81.000 \mathrm{ab}$ & $116.000 \mathrm{ab}$ & 141.667abcde & $158.000 \mathrm{abcd}$ \\
\hline M5T1 & $72.500 \mathrm{abc}$ & $94.333 \mathrm{ab}$ & $137.000 \mathrm{ab}$ & $156.333 \mathrm{abcd}$ & $173.000 \mathrm{ab}$ \\
\hline M5T2 & 71.000abcd & $103.667 \mathrm{a}$ & $143.333 \mathrm{ab}$ & $166.333 a$ & $181.667 \mathrm{a}$ \\
\hline M5T3 & $75.000 \mathrm{abc}$ & $84.333 \mathrm{ab}$ & $137.667 \mathrm{ab}$ & $158.667 \mathrm{abc}$ & $167.667 \mathrm{abcd}$ \\
\hline M6T1 & 63.333abcde & $82.333 \mathrm{ab}$ & $113.000 \mathrm{ab}$ & $126.000 \mathrm{abcde}$ & 139.333abcde \\
\hline M6T2 & $81.667 a$ & $105.667 \mathrm{a}$ & $130.000 \mathrm{ab}$ & $152.333 \mathrm{abcd}$ & $160.333 \mathrm{abcd}$ \\
\hline M6T3 & 63.000abcde & $78.667 \mathrm{ab}$ & $126.000 \mathrm{ab}$ & $151.000 \mathrm{abcd}$ & $160.000 \mathrm{abcd}$ \\
\hline M7T1 & $59.333 \mathrm{bcde}$ & $72.333 \mathrm{ab}$ & $105.333 \mathrm{ab}$ & $112.667 \mathrm{bcde}$ & $120.333 \mathrm{cde}$ \\
\hline M7T2 & 70.000abcde & $92.667 \mathrm{ab}$ & $107.000 \mathrm{ab}$ & $116.333 \mathrm{bcde}$ & 131.667abcde \\
\hline M7T3 & 64.000abcde & $91.000 \mathrm{ab}$ & $143.333 \mathrm{ab}$ & $154.000 \mathrm{abcd}$ & 161.333abcd \\
\hline F1T1 & $57.000 \mathrm{cde}$ & $70.000 \mathrm{ab}$ & $103.333 \mathrm{ab}$ & $109.667 \mathrm{de}$ & $117.667 \mathrm{de}$ \\
\hline F1T2 & $53.000 \mathrm{de}$ & $69.333 \mathrm{ab}$ & $101.667 \mathrm{ab}$ & $113.333 \mathrm{bcde}$ & $121.667 \mathrm{bcde}$ \\
\hline F1T3 & $51.000 \mathrm{e}$ & $65.000 \mathrm{~b}$ & $97.333 \mathrm{ab}$ & $124.333 \mathrm{abcde}$ & 142.333abcde \\
\hline $\begin{array}{l}\text { ANOVA based } \\
\text { on treatments } \\
\text { across weeks at } \\
\text { p }<0.05\end{array}$ & 0.000 & 0.011 & 0.000 & 0.000 & 0.000 \\
\hline $\begin{array}{l}\text { ANOVA between } \\
\text { replicates across } \\
\text { weeks at } p<0.05\end{array}$ & 0.047 & 0.156 & 0.017 & 0.292 & 0.292 \\
\hline
\end{tabular}

\section{Cost of Organic Induced Mycorrhiza Production}

Production of the organic fertilizer cost 6000 naira producing 4 bags compared to the surveyed market price of a bag of fertilizer at 8000 naira making the organic induce mycorrhiza one-fourth cheaper than an inorganic fertilizer. 
Table 3. Average mean of number of leaves from $5^{\text {th }}-9^{\text {th }}$ week of observation.

\begin{tabular}{|c|c|c|c|c|c|}
\hline & & Weeks & & & \\
\hline Treatments & $5^{\text {th }}$ & $6^{\text {th }}$ & $7^{\text {th }}$ & $8^{\text {th }}$ & 9th \\
\hline M1T1 & 7.667ab & $9.000 \mathrm{bcdef}$ & $10.333 a$ & $10.333 a$ & $10.333 \mathrm{a}$ \\
\hline M1T2 & $8.333 \mathrm{ab}$ & $10.333 \mathrm{abcd}$ & $11.000 \mathrm{a}$ & $7.000 \mathrm{a}$ & $7.000 \mathrm{a}$ \\
\hline M1T3 & 7.667ab & $10.667 \mathrm{abc}$ & $11.000 \mathrm{a}$ & $9.667 \mathrm{a}$ & $9.667 \mathrm{a}$ \\
\hline M2T1 & $8.000 \mathrm{ab}$ & $11.667 \mathrm{a}$ & $8.000 \mathrm{a}$ & $8.667 a$ & $8.667 a$ \\
\hline M2T2 & $6.000 \mathrm{~b}$ & 10.000abcde & $9.667 \mathrm{a}$ & $7.333 \mathrm{a}$ & $7.333 \mathrm{a}$ \\
\hline M2T3 & $8.333 \mathrm{ab}$ & $8.000 \mathrm{def}$ & $7.667 \mathrm{a}$ & $8.333 \mathrm{a}$ & $8.333 \mathrm{a}$ \\
\hline M3T1 & $9.000 \mathrm{a}$ & $9.000 \mathrm{bcdef}$ & $9.000 \mathrm{a}$ & $8.667 \mathrm{a}$ & $8.667 \mathrm{a}$ \\
\hline M3T2 & 7.667ab & $7.667 \mathrm{ef}$ & $11.667 \mathrm{a}$ & $8.333 \mathrm{a}$ & $8.333 a$ \\
\hline M3T3 & 7.667ab & $11.000 \mathrm{ab}$ & $10.667 \mathrm{a}$ & $8.000 \mathrm{a}$ & $8.000 \mathrm{a}$ \\
\hline M4T1 & $8.333 \mathrm{ab}$ & $10.333 \mathrm{abcd}$ & $9.000 \mathrm{a}$ & $9.667 \mathrm{a}$ & $9.667 \mathrm{a}$ \\
\hline M4T2 & $7.000 \mathrm{ab}$ & $10.667 \mathrm{abc}$ & $10.000 \mathrm{a}$ & $9.333 \mathrm{a}$ & $9.333 \mathrm{a}$ \\
\hline M4T3 & $7.000 \mathrm{ab}$ & 9.333abcdef & $8.667 \mathrm{a}$ & $9.000 \mathrm{a}$ & $9.000 \mathrm{a}$ \\
\hline M5T1 & $8.333 \mathrm{ab}$ & $10.667 \mathrm{abc}$ & $10.000 \mathrm{a}$ & $11.000 \mathrm{a}$ & $11.000 \mathrm{a}$ \\
\hline M5T2 & $6.667 \mathrm{ab}$ & $10.667 \mathrm{abc}$ & $10.000 \mathrm{a}$ & $9.333 \mathrm{a}$ & $9.333 \mathrm{a}$ \\
\hline M5T3 & $6.667 \mathrm{a} b$ & 10.000abcde & $10.667 \mathrm{a}$ & $9.000 \mathrm{a}$ & $9.000 \mathrm{a}$ \\
\hline M6T1 & $7.000 \mathrm{ab}$ & 9.667abcdef & $8.667 \mathrm{a}$ & $9.000 \mathrm{a}$ & $9.000 \mathrm{a}$ \\
\hline M6T2 & $8.333 \mathrm{ab}$ & 9.333abcdef & $9.333 \mathrm{a}$ & $9.000 \mathrm{a}$ & $9.000 \mathrm{a}$ \\
\hline M6T3 & 7.667ab & $8.333 \mathrm{cdef}$ & $9.000 \mathrm{a}$ & $8.333 \mathrm{a}$ & $8.333 \mathrm{a}$ \\
\hline M7T1 & 7.667ab & $8.667 \mathrm{bcdef}$ & $8.000 \mathrm{a}$ & $8.333 a$ & $8.333 a$ \\
\hline M7T2 & 7.333ab & $9.000 \mathrm{bcdef}$ & $8.667 \mathrm{a}$ & $8.000 \mathrm{a}$ & $8.000 \mathrm{a}$ \\
\hline M7T3 & 7.333ab & $10.667 \mathrm{abc}$ & $9.000 \mathrm{a}$ & $9.667 \mathrm{a}$ & $9.667 \mathrm{a}$ \\
\hline F1T1 & $8.000 \mathrm{ab}$ & $8.333 \mathrm{cdef}$ & $10.000 \mathrm{a}$ & $9.000 \mathrm{a}$ & $9.000 \mathrm{a}$ \\
\hline F1T2 & $8.000 \mathrm{ab}$ & $8.000 \mathrm{def}$ & $10.000 \mathrm{a}$ & $8.667 \mathrm{a}$ & $8.667 a$ \\
\hline F1T3 & $6.000 \mathrm{~b}$ & $7.333 \mathrm{f}$ & $10.000 \mathrm{a}$ & $9.000 \mathrm{a}$ & $9.000 \mathrm{a}$ \\
\hline $\begin{array}{l}\text { ANOVA based } \\
\text { on treatments } \\
\text { across weeks at } \\
\text { p }<0.05\end{array}$ & 0.006 & 0.000 & 0.081 & 0.337 & 0.337 \\
\hline $\begin{array}{l}\text { ANOVA between } \\
\text { replicates across } \\
\text { weeks at } p<0.05\end{array}$ & 0.739 & 0.405 & 0.920 & 0.896 & 0.896 \\
\hline
\end{tabular}

Findings of this research showed that organic induced mycorrhiza fertilizer has less cost implication than inorganic fertilizer. Also, mycorrhizae positively affects on plant growth by reducing germination periods and increasing the rate of plant development. Plant and mycorrhizae exudates are known to participate in complex chemical dialogues which play roles in several aspects of plant growth. Plant strigolactones function as signaling compounds during the initiation of 
Table 4. Average mean of culm diameter $[\mathrm{cm}]$ from $5^{\text {th }}-9^{\text {th }}$ week of observation.

\begin{tabular}{|c|c|c|c|c|c|}
\hline & & Weeks & & & \\
\hline Treatments & $5^{\text {th }}$ & $6^{\text {th }}$ & $7^{\text {th }}$ & $8^{\text {th }}$ & $9^{\text {th }}$ \\
\hline M1T1 & $2.000 \mathrm{a}$ & $2.667 \mathrm{a}$ & $2.667 \mathrm{a}$ & $2.667 \mathrm{a}$ & $2.667 \mathrm{a}$ \\
\hline M1T2 & $1.667 \mathrm{a}$ & $2.300 \mathrm{a}$ & $2.300 \mathrm{a}$ & $2.300 \mathrm{a}$ & $2.300 \mathrm{a}$ \\
\hline M1T3 & $1.967 \mathrm{a}$ & $2.100 \mathrm{a}$ & $2.467 \mathrm{a}$ & $2.467 \mathrm{a}$ & $2.467 \mathrm{a}$ \\
\hline M2T1 & $1.800 \mathrm{a}$ & $2.033 \mathrm{a}$ & $2.100 \mathrm{a}$ & $2.100 \mathrm{a}$ & $2.100 \mathrm{a}$ \\
\hline $\mathrm{M} 2 \mathrm{~T} 2$ & $1.933 \mathrm{a}$ & $2.600 \mathrm{a}$ & $2.800 \mathrm{a}$ & $2.867 \mathrm{a}$ & $2.867 \mathrm{a}$ \\
\hline M2T3 & $2.200 \mathrm{a}$ & $2.367 \mathrm{a}$ & $2.533 \mathrm{a}$ & $2.633 a$ & $2.633 a$ \\
\hline M3T1 & $1.700 \mathrm{a}$ & $1.833 \mathrm{a}$ & $2.000 \mathrm{a}$ & $2.000 \mathrm{a}$ & $2.000 \mathrm{a}$ \\
\hline M3T2 & $2.100 \mathrm{a}$ & $2.333 \mathrm{a}$ & $2.633 \mathrm{a}$ & $2.667 \mathrm{a}$ & $2.667 \mathrm{a}$ \\
\hline M3T3 & $1.867 \mathrm{a}$ & $2.200 \mathrm{a}$ & $2.467 \mathrm{a}$ & $2.467 \mathrm{a}$ & $2.467 \mathrm{a}$ \\
\hline $\mathrm{M} 4 \mathrm{~T} 1$ & $2.067 \mathrm{a}$ & $2.267 \mathrm{a}$ & $2.400 \mathrm{a}$ & $2.400 \mathrm{a}$ & $2.400 \mathrm{a}$ \\
\hline M4T2 & $2.000 \mathrm{a}$ & $2.600 \mathrm{a}$ & $2.633 a$ & $2.667 \mathrm{a}$ & $2.667 \mathrm{a}$ \\
\hline M4T3 & $1.800 \mathrm{a}$ & $1.833 \mathrm{a}$ & $1.833 \mathrm{a}$ & $1.833 \mathrm{a}$ & $1.833 \mathrm{a}$ \\
\hline M5T1 & $1.567 \mathrm{a}$ & $2.367 \mathrm{a}$ & $2.433 \mathrm{a}$ & $2.433 \mathrm{a}$ & $2.433 \mathrm{a}$ \\
\hline M5T2 & $1.767 \mathrm{a}$ & $2.400 \mathrm{a}$ & $2.667 \mathrm{a}$ & $2.700 \mathrm{a}$ & $2.700 \mathrm{a}$ \\
\hline M5T3 & $1.767 \mathrm{a}$ & $2.167 \mathrm{a}$ & $2.500 \mathrm{a}$ & $2.500 \mathrm{a}$ & $2.500 \mathrm{a}$ \\
\hline M6T1 & $1.667 \mathrm{a}$ & $2.067 \mathrm{a}$ & $2.167 \mathrm{a}$ & $2.233 \mathrm{a}$ & $2.233 \mathrm{a}$ \\
\hline M6T2 & $2.333 \mathrm{a}$ & $2.567 \mathrm{a}$ & $2.800 \mathrm{a}$ & $2.800 \mathrm{a}$ & $2.800 \mathrm{a}$ \\
\hline M6T3 & $1.467 \mathrm{a}$ & $2.067 \mathrm{a}$ & $2.767 \mathrm{a}$ & $2.800 \mathrm{a}$ & $2.800 \mathrm{a}$ \\
\hline M7T1 & $1.933 \mathrm{a}$ & $2.267 \mathrm{a}$ & $2.633 a$ & $2.733 \mathrm{a}$ & $2.733 \mathrm{a}$ \\
\hline M7T2 & $1.800 \mathrm{a}$ & $1.933 \mathrm{a}$ & $2.033 \mathrm{a}$ & $2.033 \mathrm{a}$ & $2.033 \mathrm{a}$ \\
\hline M7T3 & $2.233 \mathrm{a}$ & $2.467 \mathrm{a}$ & $2.867 \mathrm{a}$ & $2.867 \mathrm{a}$ & $2.867 \mathrm{a}$ \\
\hline $\mathrm{F} 1 \mathrm{~T} 1$ & $1.933 \mathrm{a}$ & $2.367 \mathrm{a}$ & $2.567 \mathrm{a}$ & $2.833 \mathrm{a}$ & $2.833 \mathrm{a}$ \\
\hline F1T2 & $1.967 \mathrm{a}$ & $2.467 \mathrm{a}$ & $2.500 \mathrm{a}$ & $2.500 \mathrm{a}$ & $2.500 \mathrm{a}$ \\
\hline F1T3 & $2.233 \mathrm{a}$ & $2.700 \mathrm{a}$ & $3.267 \mathrm{a}$ & $3.267 \mathrm{a}$ & $3.267 \mathrm{a}$ \\
\hline $\begin{array}{l}\text { ANOVA based } \\
\text { on treatments } \\
\text { across weeks at } \\
\text { p }<0.05\end{array}$ & 0.235 & 0.743 & 0.258 & 0.162 & 0.162 \\
\hline $\begin{array}{l}\text { ANOVA between } \\
\text { replicates across } \\
\text { weeks at } \mathrm{p}<0.05\end{array}$ & 0.103 & 0.665 & 0.261 & 0.183 & 0.183 \\
\hline
\end{tabular}

arbuscular mycorrhizae colonization and stimulates fungal metabolism and branching [18]. AM appears linked to increased production of auxins and cytokinins which promote plant growth [18]. 
Table 5. Average mean of leaf broadness $[\mathrm{cm}]$ from $5^{\text {th }}-9^{\text {th }}$ week of observation.

\begin{tabular}{|c|c|c|c|c|c|}
\hline & & Weeks & & & \\
\hline Treatments & $5^{\text {th }}$ & $6^{\text {th }}$ & $7^{\text {th }}$ & $8^{\text {th }}$ & $9^{\text {th }}$ \\
\hline M1T1 & $5.233 \mathrm{ab}$ & $8.167 \mathrm{a}$ & $9.000 \mathrm{a}$ & $9.000 \mathrm{a}$ & $9.000 \mathrm{a}$ \\
\hline M1T2 & 4.667ab & 7.600ab & $8.367 \mathrm{ab}$ & $8.500 a$ & $8.500 \mathrm{a}$ \\
\hline M1T3 & 6.433ab & $7.000 \mathrm{abc}$ & 7.267ab & $7.667 \mathrm{a}$ & $7.667 \mathrm{a}$ \\
\hline $\mathrm{M} 2 \mathrm{~T} 1$ & $7.067 \mathrm{a}$ & 7.667ab & 7.667ab & $8.000 \mathrm{a}$ & $8.000 \mathrm{a}$ \\
\hline M2T2 & $6.200 \mathrm{ab}$ & 7.400ab & 7.500ab & $7.667 a$ & $7.667 \mathrm{a}$ \\
\hline M2T3 & $6.867 \mathrm{a}$ & $7.800 \mathrm{a}$ & 7.867ab & $8.333 a$ & $8.333 a$ \\
\hline M3T1 & $5.667 \mathrm{ab}$ & $6.000 \mathrm{abc}$ & $6.733 \mathrm{ab}$ & $7.067 \mathrm{a}$ & $7.067 \mathrm{a}$ \\
\hline M3T2 & $6.333 \mathrm{ab}$ & $6.667 \mathrm{abc}$ & 7.000ab & $7.700 \mathrm{a}$ & $7.700 \mathrm{a}$ \\
\hline M3T3 & $4.100 \mathrm{ab}$ & $7.000 \mathrm{abc}$ & 7.467ab & $7.833 a$ & $7.833 a$ \\
\hline M4T1 & $6.033 \mathrm{ab}$ & $7.000 \mathrm{abc}$ & 7.000ab & $8.333 a$ & $7.833 a$ \\
\hline M4T2 & $4.633 \mathrm{ab}$ & 7.167ab & 7.700ab & $8.067 \mathrm{a}$ & $8.067 \mathrm{a}$ \\
\hline M4T3 & $5.000 \mathrm{ab}$ & $5.233 \mathrm{abc}$ & $6.500 \mathrm{~b}$ & $7.333 a$ & $7.333 a$ \\
\hline M5T1 & $5.333 \mathrm{ab}$ & 7.167ab & $8.600 \mathrm{ab}$ & $8.600 a$ & $8.600 \mathrm{a}$ \\
\hline M5T2 & $5.167 \mathrm{ab}$ & $6.833 \mathrm{abc}$ & $8.333 \mathrm{ab}$ & $9.000 \mathrm{a}$ & $9.000 \mathrm{a}$ \\
\hline M5T3 & $5.267 \mathrm{ab}$ & $6.500 \mathrm{abc}$ & $8.067 \mathrm{ab}$ & $8.333 \mathrm{a}$ & $8.333 a$ \\
\hline M6T1 & $5.200 \mathrm{ab}$ & 7.667ab & 7.667ab & $7.667 \mathrm{a}$ & $7.667 \mathrm{a}$ \\
\hline M6T2 & $6.500 \mathrm{ab}$ & $8.333 \mathrm{a}$ & $8.600 \mathrm{ab}$ & $8.633 a$ & $8.633 a$ \\
\hline M6T3 & $5.667 \mathrm{ab}$ & $6.700 \mathrm{abc}$ & 7.500ab & $8.167 \mathrm{a}$ & $8.167 \mathrm{a}$ \\
\hline M7T1 & $5.767 \mathrm{ab}$ & $6.667 \mathrm{abc}$ & 7.167ab & $8.000 \mathrm{a}$ & $8.000 \mathrm{a}$ \\
\hline M7T2 & $5.433 \mathrm{ab}$ & $5.933 \mathrm{abc}$ & 7.567ab & $7.567 \mathrm{a}$ & $7.567 \mathrm{a}$ \\
\hline M7T3 & $6.333 \mathrm{ab}$ & $8.333 a$ & $8.333 \mathrm{ab}$ & $8.600 a$ & $8.600 \mathrm{a}$ \\
\hline F1T1 & $5.100 \mathrm{ab}$ & $5.333 \mathrm{abc}$ & $6.833 \mathrm{ab}$ & $7.833 \mathrm{a}$ & $7.833 \mathrm{a}$ \\
\hline $\mathrm{F} 1 \mathrm{~T} 2$ & $3.000 \mathrm{~b}$ & $4.333 \mathrm{bc}$ & $6.233 b$ & $8.033 a$ & $8.033 a$ \\
\hline F1T3 & $3.333 b$ & $3.667 \mathrm{c}$ & 7.667ab & $8.967 \mathrm{a}$ & $8.967 \mathrm{a}$ \\
\hline $\begin{array}{l}\text { ANOVA based } \\
\text { on treatments } \\
\text { across weeks at } \\
\text { p }<0.05\end{array}$ & 0.005 & 0.000 & 0.004 & 0.364 & 0.364 \\
\hline $\begin{array}{l}\text { ANOVA between } \\
\text { replicates across } \\
\text { weeks at } p<0.05\end{array}$ & 0.581 & 0.515 & 0.012 & 0.245 & 0.245 \\
\hline
\end{tabular}

\section{Conclusion}

The results showed mycorrhizae induce organic fertilizer effectiveness on accelerated developmental phase of maize, cheap cost of production and little or no 


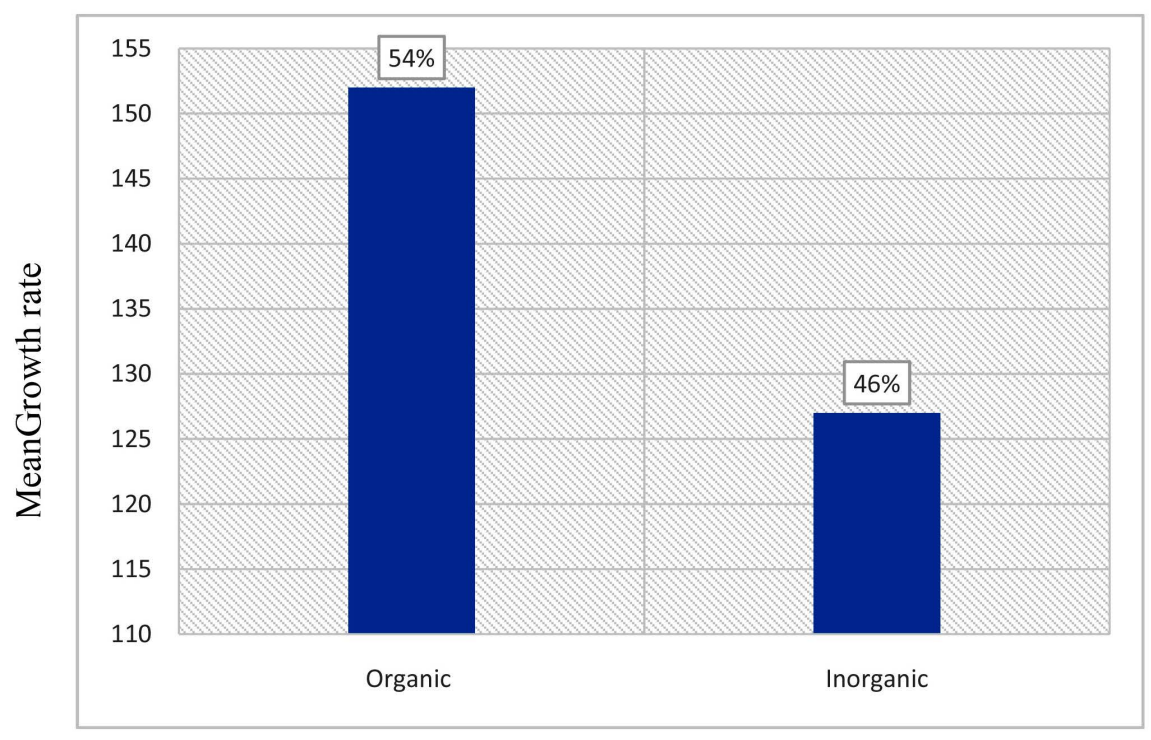

Fertilizer Type

Figure 1. Mean comparison between organic and inorganic effect on growth rate of maize.

damage on soil composition. Additionally, it can be used for colonization and propagation of desired vegetation in a natural environment and significant impact in habitat restoration efforts. Agriculture might also be positively impacted by shorter crop cycles [19].

\section{Acknowledgements}

The authors are grateful to Abubakar Tafawa Balewa University, Bauchi and IBR TETFUND REF/ATBU/DVC/Acad/12 for their financial support and also grateful to Department of Biological Sciences for providing us with the work bench and their expert support.

\section{Conflicts of Interest}

The authors declare no conflicts of interest regarding the publication of this paper.

\section{References}

[1] Adesoji, A.G. (2015) Potentials and Challenges of Inorganic and Organic Fertilizers in Nutrient Management. In: Sinha, S., Pant, S.S., Bajpai, S. and Govil, J.N., Eds., Fertilizer Technology I: Synthesis, Studium Press Llc., Houston, 77-100.

[2] Adesoji, A.G., Abubakar, I.U. and Ishaya, D.A. (2010) Crop Residue Management for Nutrient Recycling: Overview. In: Akinlade, J.A., Ogunwale, A.B., Asaolu, V.O., Aderinola, O.A., Ojebiyi, O.O. and Rafiu, T.A., Eds., Re-Strategizing Nigerian Agriculture in a Rapidly Changing Climatic Conditions for Sustainable Food Security, Proceedings of the 44th Annual Conference of the Agricultural Society of Nigeria (ASN), 18-22 October 2010, Ladoke Akintola University of Technology, Ogbomoso, Oyo State, Nigeria, 1153-1156. 
[3] CIMMYT (1988) From Agronomic Data to Farmer Recommendations: An Economics Training Manual. CIMMYT Economics Program, Mexico, 59.

[4] Fusconi, A. (2014) Regulation of Root Morphogenesis in Arbuscular Mycorrhizae: What Role Do Fungal Exudates, Phosphate, Sugars and Hormones Play in Lateral Root Formation. Annals of Botany, 113, 19-33. https://doi.org/10.1093/aob/mct258

[5] Javaid, A. (2009) Arbuscular Mycorrhizal Mediated Nutrition in Plants. Journal of Plant Nutrition, 32, 1595-1618. https://doi.org/10.1080/01904160903150875

[6] Adesoji, A.G., Abubakar, I.U. and Labe, D.A. (2015) Influence of Incorporated Legumes and Nitrogen Fertilization on Maize (Zea mays L.) Nutrient Uptake in a Semi-Arid Environment. IOSR Journal of Agriculture and Veterinary Sciences, 8, 1-8.

[7] Largent, D.L., Johnson, D. and Watling, R. (1977) How to Identify Mushrooms to Genus, Vol. III: Microscopic Features. Mad River Press, Eureka, 148.

[8] Leifheit, E.F., Veresoglou, S.D., Lehmann, A., Morris, E.K. and Rillig, M.C. (2014) Multiple Factors Influence the Role of Arbuscular Mycorrhizal Fungi in Soil Aggregation-A Meta-Analysis. Plant and Soil, 374, 523-537. https://doi.org/10.1007/s11104-013-1899-2

[9] Lombin, G. (1988) Soil Science. In: Youdeowei, A., Ezedinma, F.O.C. and Onazi, O.C., Eds., Introduction to Tropical Agriculture, Longman Group, London, 34-87.

[10] Okpanachi, I.Y. and Sahal, R.M. (2018) The Mycorrhiza Initiative: A Better Way to Manure Production for Sustainable Agriculture. In Personal Communication at Abubakar Tafawa Balewa University, Bauchi.

[11] Okpanachi, I.Y. and Sahal, R.M. (2018) The Mycorrhiza Initiative: A Better Way to Manure Production for Sustainable Agriculture.

https://www.academia.edu/37072691/

[12] Pennisi, E. (2004) The Secret Life of Fungi. Science, 304, 1620-1622. https://doi.org/10.1126/science.304.5677.1620

[13] Provost and Wallert Research (1998) Investigating the Biochemistry and Cellular Physiology of NHE1. http://www.ruf.rice.edu/ bioslabs/methods/microscopy/cellcounting

[14] Rillig, M.C., Aguilar-Trigueros, C.A., Bergmann, J., Verbruggen, E., Veresoglou, S. D. and Lehmann, A. (2015) Plant Root and Mycorrhizal Fungal Traits for Understanding Soil Aggregation. New Phytologist, 205, 1385-1388. https://doi.org/10.1111/nph.13045

[15] Rillig, M.C. and Mummey, D.L. (2006) Mycorrhizas and Soil Structure. New Phytologist, 171, 41-53. https://doi.org/10.1111/j.1469-8137.2006.01750.x

[16] Singh, A., Carsky, R.J., Lucas, E.O. and Danshiell, K. (2001) Grain Yield Response of Maize to Previous Crop and Residue Management in the Guinea Savanna of Nigeria. Proceedings of the Regional Maize Workshop, 14-18 May 2001, IITA-Cotonou, Republic of Benin, 214-224.

[17] Singh, L. (1987) Soil Fertility and Crop Yield in Savanna. Proceedings of an International Drought Symposium, Held at the Kenyatta Conference Centre, 19-23 May 1986, Nairobi, Kenya, 417-427.

[18] Valeria, G. (2015) The Effect of Mycorrhizae on Seed Germination, Development, and Reproductive Yield of Rapid Gro Radish. ESSAI, 13, 18.

[19] Verheye, W. (2010) Growth and Production of Maize: Traditional Low-Input Cultivation. In: Verheye, W.H., Ed., Land Use, Land Cover and Soil Sciences, UNESCO-EOLSS Publishers, Oxford, 1-24. http://www.eolss.net 